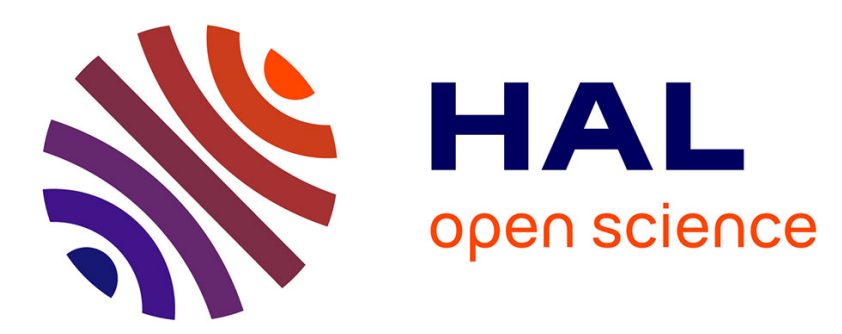

\title{
Fluctuations et comportement critique des céramiques supraconductrices à haute température critique
}

\author{
Ch. Laurent, M. Ausloos, S.K. Patapis
}

\section{To cite this version:}

Ch. Laurent, M. Ausloos, S.K. Patapis. Fluctuations et comportement critique des céramiques supraconductrices à haute température critique. Revue de Physique Appliquée, 1989, 24 (5), pp.501-506. 10.1051/rphysap:01989002405050100 . jpa-00246074

\section{HAL Id: jpa-00246074 https://hal.science/jpa-00246074}

Submitted on 1 Jan 1989

HAL is a multi-disciplinary open access archive for the deposit and dissemination of scientific research documents, whether they are published or not. The documents may come from teaching and research institutions in France or abroad, or from public or private research centers.
L'archive ouverte pluridisciplinaire HAL, est destinée au dépôt et à la diffusion de documents scientifiques de niveau recherche, publiés ou non, émanant des établissements d'enseignement et de recherche français ou étrangers, des laboratoires publics ou privés. 
Classification

Physics Abstracts

74.30G $-63.20 \mathrm{Kr}-78.30 \mathrm{Er}-63.20 \mathrm{Ls}$

\title{
Fluctuations et comportement critique des céramiques supraconductrices à haute température critique
}

\author{
Ch. Laurent $\left({ }^{1}\right)$, M. Ausloos $\left({ }^{2}\right)$ et S. K. Patapis $\left({ }^{1, *}\right)$ \\ (1) Institut Montefiore, B28, Université de Liège, B4000 Sart Tilman, Liège, Belgique \\ ${ }^{2}$ ) Institut de Physique, B5, Université de Liège, B4000 Sart Tilman, Liège, Belgique
}

(Reçu le 9 décembre 1988, révisé le 21 février 1989, accepté le 27 février 1989)

\begin{abstract}
Résumé. - Nous rappelons les résultats d'analyse de mesures précises de la résistance et du pouvoir thermoélectrique sur des polycristaux supraconducteurs de type $\mathrm{YBaCuO}$. Nous présentons une étude semblable réalisée sur des échantillons de composition $(\mathrm{BiPb}) \mathrm{CaSrCuO}$. Dans chaque cas, les méthodes permettant d'extraire des informations physiques sur la dimension effective des fluctuations supraconductrices sont discutées. Nous montrons surtout que des régimes existent conjointement dans les deux composés et représentent des comportements totalement différents.
\end{abstract}

\begin{abstract}
We refer to previous fine measurements of the electrical resistivity and of the thermoelectric power of polycristalline superconducting $\mathrm{YBaCuO}$. We present similar data on polycristalline $(\mathrm{BiPb}) \mathrm{CaSr}-$ $\mathrm{CuO}$. We discuss methods to extract physical information on the superconductivity-fluctuation dimensionality in each case. We show that similar regimes exist for both transport coefficients but differ between the $\mathrm{YBaCuO}$ and the $(\mathrm{BiPb}) \mathrm{CaSrCuO}$. Hypothesis for different behaviors are given.
\end{abstract}

\section{Introduction.}

Depuis l'annonce de la possibilité de réalisation de céramiques supraconductrices à haute température critique par Bednorz et Muller [1], de nombreux travaux ont été effectués en vue notamment d'améliorer les caractéristiques (courant critique $J_{\mathrm{c}}$, champ critique $H_{\mathrm{c}}$ ) des produits et de trouver de nouveaux composés dont la température critique $\left(T_{\mathrm{c}}\right)$ est plus élevée. Afin d'atteindre ce dernier but, il est essentiel de connaître et de comprendre les mécanismes responsables de l'apparition de la supraconductivité dans ces oxydes. C'est pourquoi de nombreuses théories ont été modifiées ou élaborées et nombre de travaux expérimentaux ont été réalisés. Parmi ceux-ci, les analyses précises des propriétés de transport présentent un grand intérêt, et ce tout particulièrement à proximité de la température critique $T_{\mathrm{c}}$. C'est ainsi qu'il est possible de mettre en évidence la dimension effective du système (caractère bi- ou tridimensionnel des fluctuations) et divers régimes de conduction.

Dans la suite, nous présentons une comparaison

(*) Adresse permanente: Solid State Div., Univ. d'Athènes, Athènes, Grèce. des résultats de mesures de la résistivité $\rho$ et du pouvoir thermoélectrique $S$ sur des matériaux frittés de type $\mathrm{Y}_{1} \mathrm{Ba}_{2} \mathrm{Cu}_{3} \mathrm{O}_{7-y}$ et $\mathrm{Bi}_{2-x} \mathrm{~Pb}_{x} \mathrm{Ca}_{2} \mathrm{Sr}_{2} \mathrm{Cu}_{3} \mathrm{O}_{10}$ $(x=0,25)$ et nous discutons quelque peu les méthodes d'analyse.

La préparation de ces échantillons est respectivement décrite dans les références [2] et [3]. On trouve également les conditions générales des mesures de résistivité et du pouvoir thermoélectrique dans les références [2] et [4].

Il est cependant important de remarquer que :

a) les mesures de résistances $R(T)$ ont été réalisées avec une densité du courant continu de l'ordre de $0,2 \mathrm{~A} / \mathrm{cm}^{2}$, le gradient sur le porte-échantillon étant réduit à une valeur inférieure à $10^{-2} \mathrm{~K} / \mathrm{cm}$ et reproductible de point à point ;

b) la méthode de mesure du pouvoir thermoélectrique $S(T)$ permet d'éviter tout effet de gradient thermique à hauteur des contacts [4].

Dans tous les cas, des précautions ont été prises afin d'éliminer l'influence des effets de redressement aux contacts (influence des signaux a.c. parasites) et la vitesse de balayage en température est faible (quelques kelvins par heure), de sorte que la sensibilité correspond à une tension de quelques $\mathrm{nV}$ aux 
bornes de l'échantillon pour les mesures des grandeurs envisagées $(R$ ou $S$ ), et à quelques $\mathrm{mK}$ en ce qui concerne la température (ou les gradients de température).

\section{Résistivité.}

Au sujet du comportement de la résistivité à proximité de $T_{c}$ en absence ou en présence de champ magnétique, on trouvera une description précise dans le cas de l'YBaCuO dans la référence [2]. On y décrit entre autres le comportement exponentiel de $R(T)$ sous $T_{\mathrm{c}}$ :

$$
R(T)=R_{\mathrm{A}} \cdot \exp \left(-\frac{T_{\mathrm{A}}-T}{T_{\mathrm{B}}}\right)
$$

où seul $T_{\mathrm{B}}$ dépend du champ. De même, la température de percolation (résistance nulle) $T_{\mathrm{R}}(\mathrm{B})$ et l'induction magnétique appliquée $B$ sont reliées simplement par la formule [2] :

$$
B \frac{\mathrm{d} T_{\mathrm{R}}}{\mathrm{d} B}=-2,9 \mathrm{~K} \text {. }
$$

Une explication est fournie par la dimension effective des chemins normaux et supraconducteurs empruntés par le courant électrique [2]. Le même type de lois a été observé sur l'échantillon de $(\mathrm{BiPb}) \mathrm{CaSrCuO}$.
Une hystérèse anormale sous $T_{\mathrm{c}}$ en présence d'une faible induction magnétique (inférieure à $4000 \mathrm{G}$ ) lorsque l'on effectue des balayages successifs à température décroissante $(R \downarrow)$ et croissante $(R \uparrow)$ a été décelée dans le cas de l'YBaCuO $(R \uparrow>R \downarrow)$ et n'a pu être mise en évidence sur le composé au bismuth $[2,5]$. Cette différence de comportement est explicable soit par la faiblesse, dans le second échantillon, du champ critique inférieur et des forces d'ancrage des vortex à proximité de $T_{\mathrm{c}}$, soit par une position différente des «liens faibles» au sein du matériau. On peut en effet imaginer qu'ils sont plus nombreux dans les grains du ( $\mathrm{BiPb}) \mathrm{CaSrCuO}$.

Signalons également l'anisotropie induite dans un matériau polycristallin uniforme par la présence d'un champ magnétique et l'importance de connaître la géométrie utilisée [6].

En ce qui concerne l'YBaCuO, diverses analyses expérimentales de la résistivité $\rho$ ont été réalisées afin de déterminer la dimension des fluctuations supraconductrices sur des échantillons frittés [7, 8]. Dans tous les cas, l'étude repose sur la caractérisation du comportement de l'excès de conductivité $\Delta \sigma$ :

$$
\Delta \sigma=\sigma-\sigma_{\mathrm{o}},
$$

où $\sigma=1 / \rho$ est la conductivité observée, et $\sigma_{\mathrm{o}}$ est la " conductivité normale » obtenue par prolongement de la loi observée à température ambiante (où

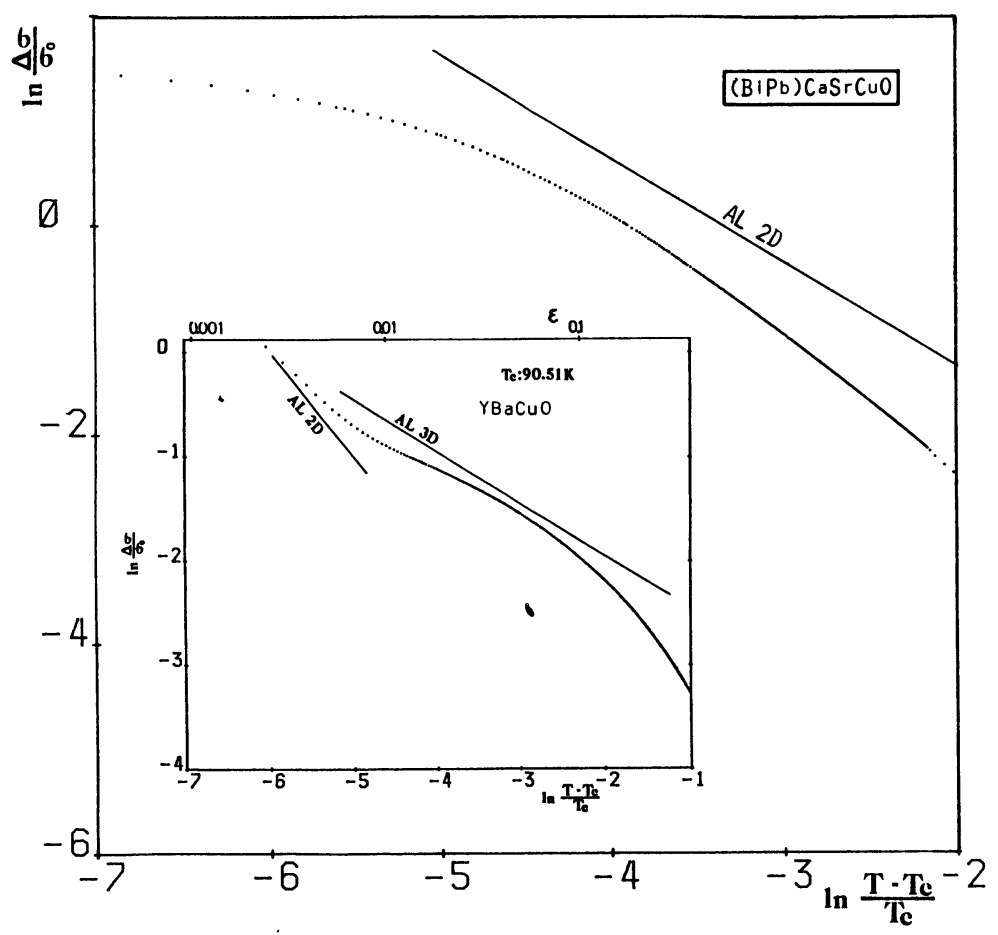

Fig. 1. - Diagramme log-log de l'excès de conductivité $\Delta \sigma$ en fonction de la température réduite $\varepsilon=\left(T-T_{\mathrm{c}}\right) / T_{\mathrm{c}}$. Les droites ont la pente théorique prédite par Aslamazov et Larkin dans les cas bi- et tridimensionnels (2D et 3D).

[Log-log plot of the «excess conductivity » $\Delta \sigma$ as a function of the reduced temperature $\varepsilon=\left(T-T_{\mathrm{c}}\right) / T_{\mathrm{c}}$. Theoretical slopes of Aslamazov-Larkin theory are given (bi- and tridimentionality cases).] 
$\rho(T)$ suit une loi linéaire). Malheureusement, la recherche de divers régimes de conductivité et leur délimitation par cette méthode est à tout le moins ambiguë. Ainsi, l'encart de la figure 1 compare, dans le cas de l'YBaCuO le comportement de $\Delta \sigma$ au modèle théorique de Aslamazov et Larkin $[9$, 10]. La droite théorique (AL3D) n'est apparemment qu'une tangente à la courbe des résultats ; ceci ne peut permettre de conclure à la présence de fluctuations tridimensionnelles (déduites sur base de mesures moins précises dans l'article de référence de Freitas et al. [7]). Cette même difficulté d'interprétation sur base exclusive des mesures de $\Delta \sigma$ se retrouve également dans le cas du composé au bismuth (Fig. 1). Il y a lieu de signaler également que la comparaison des résultats, sans paramètre d'ajustement, avec le modèle théorique d'Aslamazov et Larkin [10] pour des fluctuations bidimensionnelles nécessite la connaissance de la conductivité dans le plan $(a, b)$ de ces composés fortement anisotropes, comme nous l'avons fait remarquer dans la référence [9].

L'acquisition d'un grand nombre de mesures précises a déjà permis une analyse différente par l'intermédiaire de l'étude de la résistivité de l'YBaCuO [9] (cf. Fig. 2 encart). Cette approche présente divers avantages: a) l'utilisation de la dérivée met en exergue le terme le plus divergent à $T_{\mathrm{c}}$; b) l'incertitude quant à la vraie valeur à attribuer à $\sigma_{\mathrm{o}}$ joue un rôle réduit. En outre, cette méthode a permis de déterminer des lois très simples rendant compte du comportement de $\rho(T)$. Les domaines respectifs de validité de ces lois sont également clairement définis. Ainsi, la dérivée de la résistivité portée sur un diagramme log.-log. en fonction de la température réduite $\varepsilon=\left(T-T_{\mathrm{c}}\right) / T_{\mathrm{c}}$ montre une bonne correspondance avec un modèle de fluctuations bidimensionnelles à proximité de $T_{\mathrm{c}}\left(\varepsilon<10^{-2}\right)$, au sens de la théorie de Aslamazov et Larkin [9, 10] (Fig. 2 encart) :

$$
\frac{\mathrm{d} \rho}{\mathrm{d} T}=A \cdot \varepsilon^{-2}+B
$$

A plus haute température (Fig. 2 encart), un comportement logarithmique non prédit par Aslamazov et Larkin [10] est vérifié jusque $T \simeq 2 T_{\mathrm{c}}$ (gamme de température caractérisée par plusieurs régimes dans la référence [8]). Ce résultat correspond bien à l'observation d'un comportement logarithmique de l'excès de la conductivité dans le plan $(a, b)$ sur un monocristal [11] (mais ce, seulement jusque $\varepsilon \cong 0,2$ ). L'explication de ce comportement pourrait dès lors résider dans un processus de «brisure » de paires décrit par Maki et Thompson [11, 12].

Un même type d'analyse répété dans le cas de l'échantillon au bismuth permet à nouveau d'observer deux régimes et notamment un comportement particulier aux «températures élevées », jusque

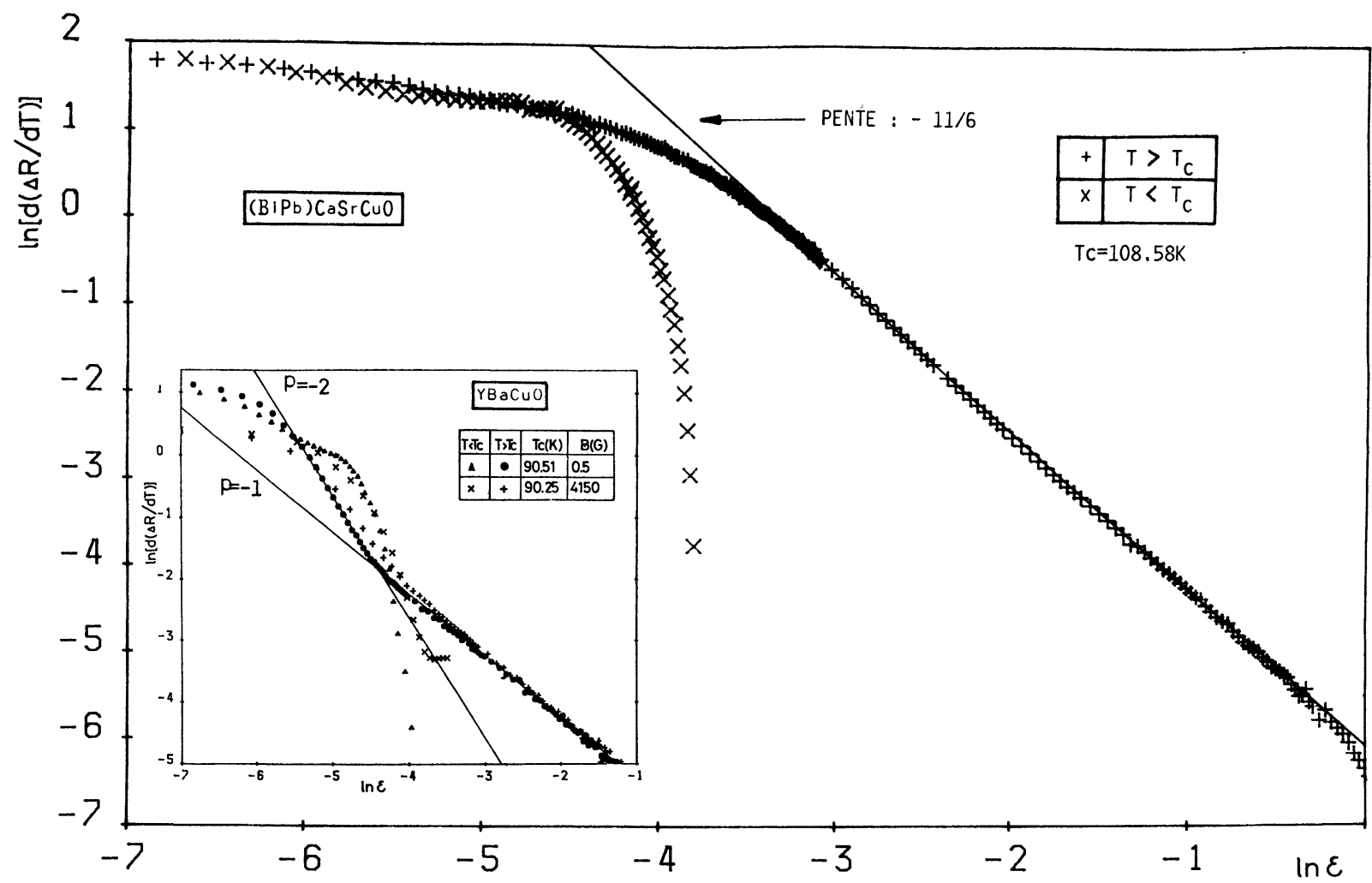

Fig. 2. - Diagramme log-log de la dérivée de l'excès de résistance en fonction de la température réduite $\varepsilon$.

[ $\log -\log$ plot of the temperature derivative of the « excess resistivity » as a function of reduced temperature.] 
$T \simeq 2 T_{\text {c }}$ (Fig. 2). La pente de la droite sur le diagramme log.-log. est beaucoup plus importante que celle présentée par l'YBaCuO et est traduite par une loi en puissance qui ne correspond apparemment pas à des modèles théoriques existants :

$$
\frac{\mathrm{d} \rho}{\mathrm{d} T}=C \cdot \varepsilon^{-11 / 6}+D .
$$

A proximité de $T_{\mathrm{c}}\left(\varepsilon<1,5 \times 10^{-2}\right)$, la pente sur le diagramme log.-log. diminue (Fig. 2) en raison soit $\mathrm{du}$ caractère tridimensionnel des fluctuations dans cette région, soit à cause d'une distribution des températures critiques au sein de la phase responsable de la transition à $110 \mathrm{~K}$ [3, 13]. Il est donc nécessaire d'affiner la préparation de la phase à $110 \mathrm{~K}$ avant de pouvoir tirer une conclusion définitive.

\section{Pouvoir thermoélectrique.}

L'interprétation des résultats des mesures de pouvoir thermoélectrique $S(T)$ est beaucoup plus difficile et ne permet pas de tirer une conclusion simple quant au signe des porteurs de charges [14]. D'autre part, les mesures sont toujours moins précises que celles de la résistivité et le gradient de température présent sur l'échantillon engendre un processus de moyenne dont il y a lieu éventuellement de tenir compte lors de l'analyse des résultats à proximité de $T_{\mathrm{c}}[4,15]$.

La valeur et même le signe du pouvoir thermoélectrique dans ce type de matériau est fortement tributaire de la composition (taux d'oxygène) et du mode de préparation [16]. La mesure de cette grandeur pourrait donc être envisagée comme un test rapide de la qualité d'échantillons.
Sur la figure 3 , on présente les résultats obtenus sur les échantillons envisagés ci-dessus. En ce qui concerne l'YBaCuO, en présence de champ magnétique, on retrouve une structure « en pied » semblable à celle observée pour la résistance [4].

Par contre, dans le cas du ( $\mathrm{BiPb}) \mathrm{SrCaCuO}$, on remarque clairement une inversion du signe du pouvoir thermoélectrique $S(T)$ sous la température critique $T_{\mathrm{c}}$, en présence et en absence de champ magnétique (Fig. 3). Ce phénomène est explicable aisément par la présence dans l'échantillon de zones dont le pouvoir thermoélectrique est positif $\left(S_{\mathrm{A}}>0\right)$ et dont la transition supraconductrice s'effectue vers $109 \mathrm{~K}\left(S_{\mathrm{A}}\left(T<T_{\mathrm{c}}\right)=0\right)$ et d'une seconde partie $\mathrm{B}$ de l'échantillon dont la contribution globale est négative $\left(S_{\mathrm{B}}<0\right)$ au moins jusqu'à une température inférieure à $80 \mathrm{~K}$. Quant à la nature de la partie $\mathrm{B}$, on peut la comprendre à partir de plusieurs hypothèses, p. ex. : a) l'échantillon est polyphasé [3] ; b) l'orientation de chaque cristal par rapport au gradient thermique introduit des contributions de signes opposés, puisque les éléments du tenseur $S$ peuvent avoir des signes différents dans un composé anisotrope (et une valeur non nulle de $R$ et de $S$ peut exister sous $T_{\mathrm{c}}$ dans la direction perpendiculaire aux plans supraconducteurs) ; c) la résistance et le pouvoir thermoélectrique des zones de liaisons faibles dépendent de leur composition chimique qui peut être différente des concentrations volumiques. Une connaissance plus approfondie de la structure et de la texture de ce type d'échantillons et les résultats d'expériences sur des monocristaux devraient permettre d'élucider ce problème. Il est cependant important de remarquer que dans l'air à température ambiante et au-delà, une dégradation des échantil-

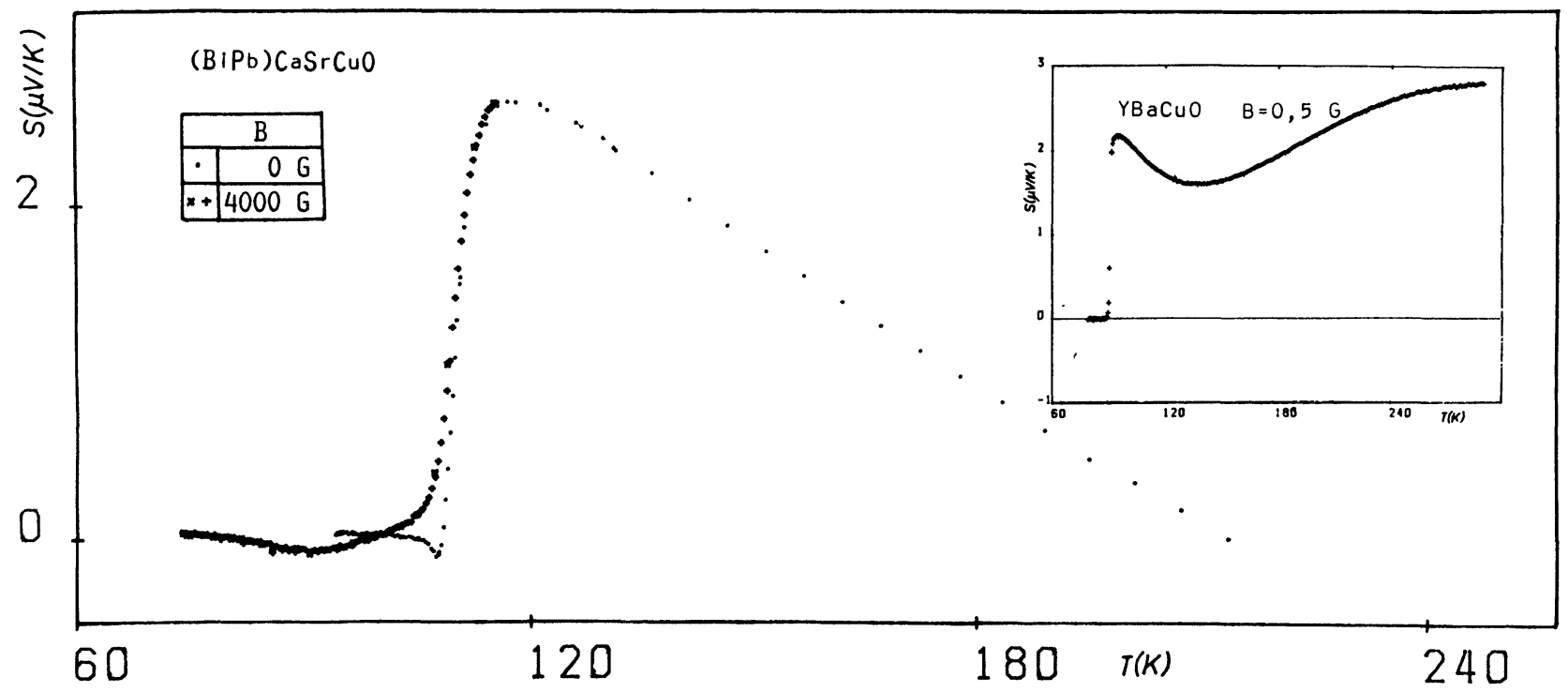

Fig. 3. - Allure du pouvoir thermoélectrique en fonction de la température.

[Behaviour of thermoelectric power $S$ as a function of temperature.] 


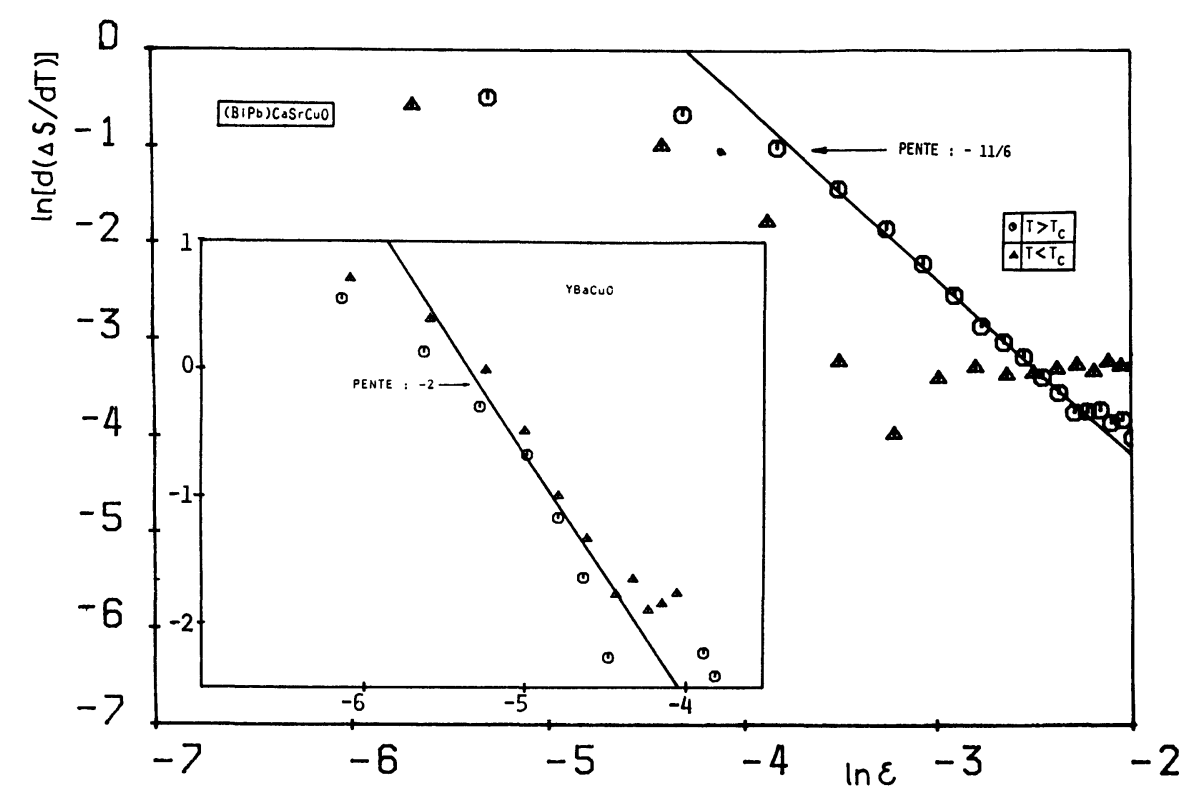

Fig. 4. - Diagramme log-log de la dérivée de l'excès du pouvoir thermoélectrique $S(T)$ en fonction de la température réduite $\varepsilon$.

[Temperature derivative of the « excess thermoelectric power » as a function of reduced temperature on a log-log plot.]

lons se produit avec une diminution de la valeur du pouvoir thermoélectrique (augmentation de la contribution de la partie B), conjointement à une augmentation de l'amplitude de la portion négative sous $T_{\mathrm{c}}$.

En raison de l'imprécision sur les mesures du pouvoir thermoélectrique, on ne peut analyser les fluctuations que dans la région où les variations en fonction de la température sont importantes. Pour les deux composés, dans une zone proche de $T_{\mathrm{c}}$, on observe un comportement de $S(T)$ identique à celui de $R(T)$, dans les mêmes intervalles de température (Fig. 4), ce qui correspond bien aux prédictions théoriques [17].

\section{Conclusions.}

Nous avons montré qu'une étude précise de la dérivée des grandeurs $R(T)$ et $S(T)$ permet de déterminer des lois représentatives du comportement des fluctuations supraconductrices.

La présence de régimes caractéristiques a été mise en évidence dans le cas des céramiques supraconductrices de composition $\mathrm{Y}_{1} \mathrm{Ba}_{2} \mathrm{Cu}_{3} \mathrm{O}_{7-y}$ et $\mathrm{Bi}_{1.75} \mathrm{~Pb}_{0.25} \mathrm{Ca}_{2} \mathrm{Sr}_{2} \mathrm{Cu}_{3} \mathrm{O}_{10}$. La différence de comportement de ces deux composés est importante.

Les exposants critiques sont clairement définis pour $\varepsilon>3 \times 10^{-2}$ dans le cas du composé au Bi et pour $\varepsilon>5 \times 10^{-3}$ dans l'YBaCuO. Malgré la précision des mesures, la région $\varepsilon \rightarrow 0$ ne peut être analysée rigoureusement en raison de l'inhomogénéité dans ces matériaux (il existe sans aucun doute une distribution de $T_{\mathrm{c}}$ ).

La nécessité de l'élaboration de modèles théoriques rendant compte des phénomènes observées a été mise en exergue.

La présence de contributions de signes contraires sur les résultats de mesures du pouvoir thermoélectrique dans le cas du composé au bismuth a été démontrée.

Des mesures précises de $R(T)$ et de $S(T)$ sur des monocristaux assureront un progrès certain dans la compréhension de tous les phénomènes reportés cidessus.

\section{Remerciements.}

Nous tenons à remercier le Pr. Tarte P., le Dr. Rulmont A. (université de Liège), les Prs Green S.M., Luo H. M. (San Diego University) et le Pr. Politis C. (Kernforschungszentrum, Karlsruhe) pour la préparation des échantillons.

Les présentes recherches ont bénéficié du support financier du F.N.R.S. (Fonds National de la Recherche Scientifique, Belgique). 


\section{Bibliographie}

[1] Bednorz J. G., Muller K. A., Z. Phys. B 64 (1986) 189.

[2] Laurent Ch., Laguesse M., Patapis S. K., VanDerschueren H. W., Lecomte G. V., Rulmont A., Tarte P., Ausloos M., Z. Phys. B 69 (1988) 435.

[3] Green S. M., Jiang C., Mei Yu, Luo H. L., Politis C., Phys. Rev. B 38 (1988) 5016.

[4] Laurent Ch., Patapis S. K., Vanderschueren H. W., Rulmont A., Tarte P., Ausloos M., Solid State Commun. 66 (1988) 445.

[5] Ausloss M., Laurent Ch., Patapis S. K., RulMONT A., TARTE P., Mod. Phys. Lett. B 3 (1989) 167.

[6] Laurent Ch., Vanderschueren H. W., Tarte P., Rulmont A., Ausloos M., Appl. Phys. Lett. 52 (1988) 1179.

[7] Freitas P. P., Tsuei C. C., Plaskett T. S., Phys. Rev. B 36 (1987) 833.

[8] Vidal F., Veira J. A., Maza J., Garcia-Alvarado, Moran E., Alario M. A., J. Phys. C 21 (1988) L59.
[9] Ausloos M., Laurent Ch., Phys. Rev. B 37 (1988) 611.

[10] Aslamazov L. G., Larkin A. I., Phys. Lett. A 26 (1988) 238.

[11] Fiory A. T., Hebard A. F., Schneemeyer L. F., WASzcZAK J. V., Mat. Res. Soc. Boston (1987).

[12] Thompson R. S., Phys. Rev. B 1 (1970) 327.

[13] Politis C., Appl. Phys. A 45 (1988) 261.

[14] Ausloos M., Durczewski K., Patapis S. K., LAURENT Ch., VANDERSChUERen H. W., Solid State Commun. 65 (1988) 365.

[15] Laurent Ch., Thèse de doctorat, Univ. de Liège (1988).

[16] Henkie Z., Markowski P. J., Horyn R., Bukowski Z., Klamut J., Phys. Status Solidi B 146 (1988) K131.

[17] Ausloos M:, Magnetic Phase Transitions, Eds. M. Ausloos et R. J. Elliott (Springer, Berlin) 1983, pp. 99-129. 